\title{
Impact of edge shape on the functionalities of graphene-based single-molecule electronics devices
}

\author{
D. Carrascal, ${ }^{1,2}$ V. M. García-Suárez, ${ }^{1,2,3}$ and J. Ferrer ${ }^{1,2,3}$ \\ ${ }^{1}$ Departamento de Física, Universidad de Oviedo, 33007 Oviedo, Spain \\ ${ }^{2}$ Nanomaterials and Nanotechnology Research Center, CSIC - Universidad de Oviedo, Spain \\ ${ }^{3}$ Department of Physics, Lancaster University, Lancaster LA1 4YW, United Kingdom
}

(Received 13 February 2012; published 16 May 2012)

\begin{abstract}
We present an ab initio analysis of the impact of edge shape and graphene-molecule anchor coupling on the electronic and transport functionalities of graphene-based molecular electronics devices. We analyze how Fano-like resonances, spin filtering, and negative differential resistance effects may or may not arise by modifying suitably the edge shapes and the terminating groups of simple organic molecules. We show that the spin filtering effect is a consequence of the magnetic behavior of zigzag-terminated edges, which is enhanced by furnishing these with a wedge shape. The negative differential resistance effect is originated by the presence of two degenerate electronic states localized at each of the atoms coupling the molecule to graphene which are strongly affected by a bias voltage. The effect could thus be tailored by a suitable choice of the molecule and contact atoms if edge shape could be controlled with atomic precision.
\end{abstract}

DOI: 10.1103/PhysRevB.85.195434

PACS number(s): 31.15.A-, 73.23.Ad, 73.63.Fg, 72.80.Ga

\section{INTRODUCTION}

Graphene, which is a monolayer of carbon atoms arranged in a honeycomb lattice, has unique properties excellently matching the requirements for ideal electrodes: it is an extraordinary conductor, it is mechanically extremely robust and chemically very stable, and it withstands electro-migration. Recent advances in graphene nanoribbon fabrication and patterning $^{1-4}$ have reinforced the plausibility of the use of graphene $^{5-7}$ as a future nanoelectronics technology. The deployment of graphene nanoelectronics should have inherent advantages, since the unique two-dimensional nature of graphene should enable the fabrication and visualization of stable nanometer-sized devices of controlled shape.

The persistent inability to visualize and control the shape of bulky noble metal electrodes at the atomic scale, as well as the positioning of molecules inside the physical gap, are hindering the blossoming of molecular electronics as a mature science and its deployment as a technology. Graphene's flat nature should enable the imaging and control of the features of a single-molecule junction with atomic precision via scanning tunneling microscopy techniques. This could allow the detailed characterization of the junctions by correlating the experimentally determined atomic arrangement with the electrical properties and with the results of theoretical calculations. In addition, using molecules to bridge graphene nanojunctions should furnish the array of expected applications of graphene electronics with a whole suite of new functionalities. ${ }^{8-10}$

A crucial pending issue for the use of graphene nanoelectronics is the difficulty in controlling the edges of graphene sheets. This could not only influence the electronic properties of the graphene electrodes but it could also change arbitrarily the coupling and switching functionality of the molecules attached. However, controlled formation of sharp zigzag and armchair edges has been achieved. ${ }^{11}$ Furthermore, nanogap electrodes with gap size below $10 \mathrm{~nm}$ have been recently demonstrated, together with gating of single- or fewmolecule junctions. ${ }^{12}$ Other approaches that could lead to the controlled formation of nanogap graphene electrodes include atomic force microscopy nanolithography of graphene ${ }^{13}$ and atomically precise fabrication of graphene nanoribbons by on-surface synthesis methods. ${ }^{14}$

The tiny size of the currents involved in single- or few-molecule electronic junctions renders measuring them a difficult task. Various solutions have been proposed to solve this problem, but until now none of them have been thoroughly tested. The small size of the current is mainly a consequence of the mismatch between the on-site energy levels of the molecule and of the metallic electrodes, which reduces the coupling between them and therefore decreases the width of the transport resonances. A simple alternative would therefore consist of choosing electrodes with on-site levels that match the molecular ones, since this would enhance the transmission through the system and improve the transport properties. Graphene seems the perfect candidate because carbon is the main ingredient in most molecular compounds.

We analyze here a series of molecular junctions whose electrodes are graphene sheets terminated in a wedge geometry with either armchair, zigzag, or mixed edges as sketched in Figs. 1-3, which could mimic the nanogap junctions fabricated in Ref. 12. We also mention that controlled fabrication of the mixed wedges shown in Fig. 2 has been demonstrated in Ref. 11. To simplify matters, the geometry of the proposed junctions is such that the tips of the two wedges face opposite to each other. We have simulated the wedges at two different separations, which are also shown in Figs. 1-3. The different shapes and distances enable the molecules to position themselves at the most favorable energy minima. The tip-to-tip distance must be tailored to be of the order of, or somewhat smaller than, the length of the molecule, because a single molecule of length $L$ can only bridge the two graphene wedges provided that the two tips are positioned at a distance $d$ equal to or slightly smaller than $L$.

The aim of this article is to show that the edge shape determines the functionality of single-molecule graphene junctions. To convey this message, we have chosen two simple molecules to bridge the graphene electrodes. The first is benzene-dithiolate (BDT, S- $\mathrm{C}_{6} \mathrm{H}_{4}-\mathrm{S}$ ), which is used to fabricate the paradigmatic gold-BDT-gold molecular junction. The second molecule is bipyridine (BPD, N-C $\mathrm{CH}_{4}-\mathrm{N}$ ), where 

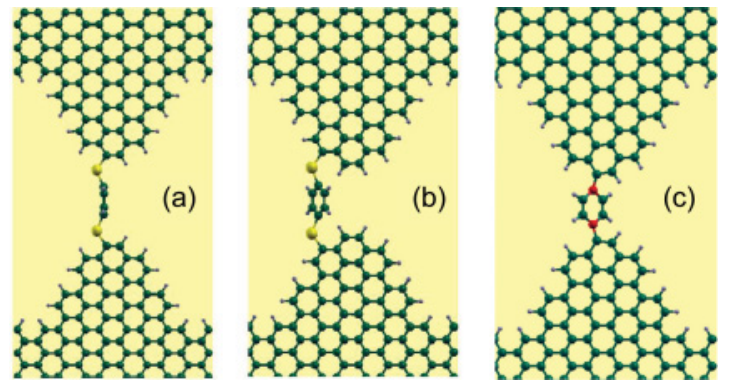

FIG. 1. (Color online) Schematic view of an armchair wedge junction bridged by a BDT molecule, where the tips are separated by (a) $9.4 \AA$ and (b) $7.1 \AA$, and by a BPD molecule where the tips are separated by $6.5 \AA$ (c). The distance is measured between the last carbon atoms at both wedges.

the S-C dimers at the edges of the BDT molecule have been replaced by a nitrogen atom. However, we stress again that the main message of the present paper relates to the edge shape and is fairly independent of the specific element chosen to bind to the carbon atoms at the graphene edges. The rationale guiding our choice is as follows. First, BDT is a reference system in molecular electronics. ${ }^{15-18}$ Because BDT and BPD are very simple molecules, gold-BDT(BPD)gold junctions do not show any functionality. Therefore, the functionalities that arise in the present graphene junctions can unambiguously be attributed to the shape of graphene edges. Second, these two molecules do not provide a good electrical connection to gold electrodes because the coupling atoms (sulfur and nitrogen, respectively) have on-site energies which are too different from those at the noble-metal electrodes and therefore introduce energy barriers between the central molecular backbone and the electrode's conducting channels. These barriers decrease the conductance through the system. We wish to check whether similar energy barriers appear when graphene is used as the electrode of choice. Third, graphitic structures have been doped with $\mathrm{B}, \mathrm{N}, \mathrm{P}, \mathrm{S}$, and $\mathrm{Si}$, where these elements form permanent covalent bonds with the matrix of carbon atoms. ${ }^{19,20}$ We therefore expect that sulfur- and nitrogen-capped molecules could attach to graphene edges making stable junctions. One of the secondary objectives of this article is therefore to check for the formation and stability of graphene-BDT(BPD)-graphene junctions.

We find that even these two simple molecules can lead to a large variety of nontrivial functionalities provided a suitable wedge is chosen. Specifically, we show here that some

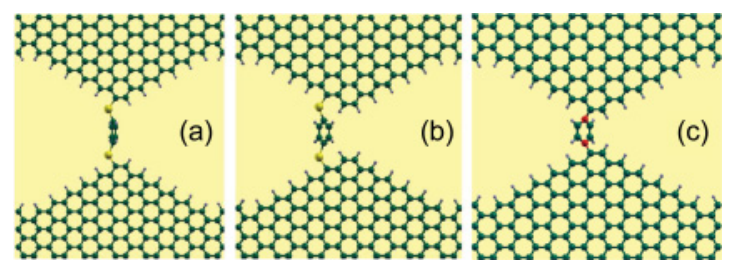

FIG. 2. (Color online) Schematic view of a mixed zigzagarmchair wedge junction bridged by a BDT molecule, where the tips are separated by (a) $9.5 \AA$ and (b) $7.2 \AA$, and by a BPD molecule, where the tips are separated by $6.5 \AA$ (c). The distance is measured between the last carbon atoms at both wedges.
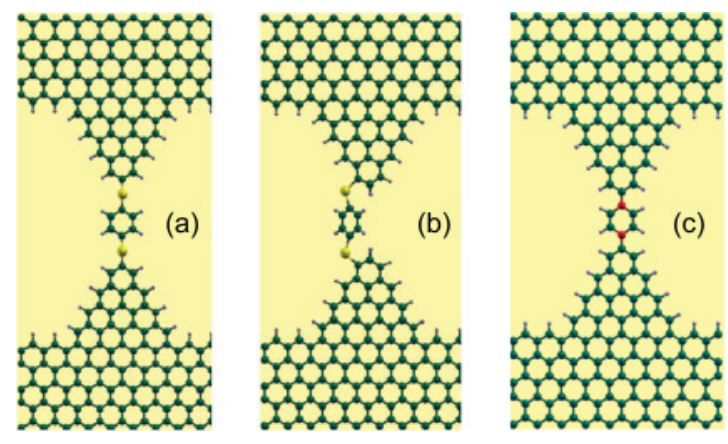

FIG. 3. (Color online) Schematic view of a zigzag wedge junction bridged by a BDT molecule, where the tips are separated by (a) $9.6 \AA$ and (b) $8.2 \AA$, and by a BPD molecule where the tips are separated by $7 \AA$ (c). The distance is measured between the last carbon atoms at both wedges.

graphene-wedge BDT and BPD junctions show negative differential resistance (NDR), ${ }^{21}$ spin-filtering (SP) behavior, ${ }^{22,23}$ and Fano resonances ${ }^{24}$ We mention that nontrivial functionalities such as NDR, ${ }^{25,26}$ switching behavior, ${ }^{27}$ or spin-filtering effects $^{28,29}$ in graphene-based atomic or molecular junctions have actually been predicted in the recent past. We offer here a comprehensive analysis with differently terminated wedges, several junction gap lengths, and two different molecules. The large phase space analyzed allows us to understand better how these diverse functionalities could be tailored on demand if or when graphene edge shapes could be controlled with atomic precision.

The SP effect can be seen when magnetic molecules join two paramagnetic noble metal electrodes. This effect has been predicted to appear when $\mathrm{Mn}_{12}$ molecular magnets bridge gold electrodes. ${ }^{22,23}$ It originates because the nanojunction allows the passage of only one of the two spin components from one side to the other. Spintronics phenomena in graphenebased electronics were also proposed some time ago $^{30,31}$ because zigzag edges or nanoislands either unpassivated or passivated with a single hydrogen atom per carbon atom are magnetic. ${ }^{32,33}$ However, the lowest energy configuration at ambient conditions for straight zigzag edges contains alternating single- and double-hydrogen bonded carbon atoms, and the resulting structure is nonmagnetic. ${ }^{34}$

The NDR effect is also sought after at the nanoscale due to its wide variety of uses and applications such as in digital-to-analog converters, oscillators, rectifiers, and amplifiers. ${ }^{35}$ An efficient NDR device is expected to display $I-V$ characteristics featuring a sharp current peak at low bias voltages, immediately followed by a low current minimum. Several mechanisms producing the NDR effect have been proposed. In molecules between silicon electrodes, it is generated by the motion of resonances toward the silicon gap; ${ }^{36}$ this motion reduces the transmission through the system and decreases the current. Another possible mechanism is a voltage-driven energy mismatch of molecular levels which leads to the destruction of resonances. ${ }^{37-40}$ The specific NDR effect demonstrated in this paper is originated by the presence of pairs of electronic states placed close to the Fermi level, and localized close to the molecule-graphene contacts. Each of these two states moves in opposite energy directions under 
the application of a bias voltage. This produces a reduction of the width and height of the corresponding resonances in the transmission coefficients. Therefore, for a given voltage the current decreases, and the NDR effect arises. In contrast, states that are localized in the middle of the molecule are not so much affected by the bias voltage.

The layout of the article is as follows: Details of the DFT theoretical methods and simulations are presented in Sec. II. Transport through BDT and BPD molecules is analyzed in Secs. III and IV, respectively. The article closes with a discussion in Sec. V. A simple model which explains the graphene-wedge NDR behavior found in the wedge junctions presented here is included in the Appendix.

\section{THEORETICAL METHOD}

The $a b$ initio simulations have been performed with the density functional theory (DFT) code SIESTA, ${ }^{41}$ which uses norm-conserving pseudopotentials to get rid of the core electrons, and pseudoatomic orbitals in the basis set to span the valence states. We have used in this case an optimized double- $\zeta$ basis set, which is enough to describe accurately the graphene band structure. We have chosen the exchange and correlation potential in the local density approximation (LDA) as parametrized by Ceperley and Alder, ${ }^{42}$ but we expect that our results should be robust enough to withstand the use of more accurate functionals describing better the van der Waals interaction. $^{43}$ The density, the Hamiltonian, and the overlap matrix elements have been calculated in a real space grid defined with a cutoff of $450 \mathrm{Ry}$. The structural relaxations of the junctions have been obtained using a single $k$ point which is enough to converge those properties. All forces have been relaxed up to a tolerance smaller than $0.001 \mathrm{eV} / \AA$. A correct analysis of the electronic structure including the density of states $($ DOS) has required a $30 \times 30 k$ grid.

Portions of the simulation cells are sketched in Figs. 1-3. The graphene sheets extend in the $X Z$ plane, with the wedges and nanojunctions oriented along the $Z$ axis and the sheet width extended along the $X$ axis. We have used periodic boundary conditions along the three spatial directions, so that graphene sheets in neighboring simulation cells are connected along the $X$ and $Z$ directions to avoid the presence of additional edges that would distort the electronic structure. We have measured sheet lengths counting the number $N$ of dimer/zigzag lines for the armchair/zigzag direction, following the convention for graphene nanoribbon unit cells. The simulated sheets have a width $N$ in the range 10-20. Along the transport direction, they have three unit cells, corresponding to $N=6$. We have then appended the wedges, whose edges have been passivated with a hydrogen atom per carbon atom.

The transport calculations have been carried out with the aid of our nonequilibrium transport code SMEAGOL. ${ }^{44}$ The system has been divided in three pieces: left lead, right lead, and extended molecule. The leads' transport channels impinging from each lead onto the molecule have been determined with a previous calculation for each different lead. These lead calculations require simulating a bulk unit cell with nearest-neighbor coupling and periodic boundary conditions. The lead's electronic structure must be converged using a sizable number of $k$ points along the direction parallel to the electronic transport. The lead's calculations serve to determine the self-energies of the electrodes and to ensure that the electronic structure at the two edges of the junction agree with those of a bulk lead.

The extended molecule includes the central part of the junction (molecule attached to graphene wedges) and also some layers of the graphene leads to further ensure that the electronic structure at the edges agrees with the lead's electronic structure. The atomic coordinates must match those of the leads. Furthermore, basis functions and accuracy parameters must also be consistent with those of the lead's calculations. The code computes the electronic structure using the nonequilibrium Green's functions formalism. We have used in the present calculation $10 k$ points in the direction transverse to the electronic transport, and we have converged the density matrix down to a tolerance smaller than $5 \times 10^{-5}$. We have extracted the transmission coefficients of the junctions and their current at the end of each simulation.

\section{WEDGE-BDT-WEDGE JUNCTIONS}

We describe first the details of the force relaxation simulations of the wedge junctions bridged by BDT molecules. We have simulated first fully passivated wedges. For each given simulation, we have placed initially a single benzenedithiolated molecule close to the two tips, and then we relaxed the forces. Most of these simulations end with a fully developed junction. Both the graphene wedges and the molecules are slightly bent or deformed at the end of the simulations to accommodate for their chemical bonding. Further, we have performed simulations that include two molecules to check the rate of single versus double molecule junctions. Indeed, both single or double junction formation are achieved when the two molecules are initially placed at opposite sides of the tips. However, we have found that if the molecules are placed initially not too far away from each other, the sulfur atoms at the two molecules rather bind to each other and no junction is developed. We are led to conclude that junction formation is highly implausible for fully passivated wedges and thiolated molecules, since the molecules bind to each other before reaching the junction area. To fix this problem, we have substituted the thiolate by thiol end groups at the sides of the molecules, We have checked that thiol-capped molecules do not bind to each other, but their reactivity is so reduced that they do not bind to the graphene edges either. We have indeed been unable to achieve the formation of a single junction in any of the simulations carried out.

We have decided as a consequence to passivate the wedges only partially, so that one or several of the passivating hydrogen atoms in the vicinity of the tip area are missing, and to cap the molecules with thiol, instead of thiolated, end groups to avoid the chemical bonding between any two of them. We have found that the edge areas containing an unpassivated carbon and its nearest-neighbor carbon atoms are extremely reactive. When the molecules reach the unpassivated area, their sulfur atoms are stripped of their hydrogen terminations. Molecule-wedge chemical bonds are hence formed, where the sulfur atoms are bound to the previously unpassivated carbon atoms, and the stripped hydrogens are attached to other nearby carbon atoms at the graphene edge. Further, the hydrogen affinity of the edges 
is so large that in some cases the inner carbon atoms of some of the molecules are also stripped of their hydrogen atoms, which end up attached to other previously unpassivated edge carbon atoms. We have performed simulations with unpassivated areas at both wedges. We have found that junctions are made only when the distance $d$ between the unpassivated carbons is approximately equal to the length $L$ of the molecule. Those simulations where $d$ is larger or considerably shorter than $L$ end up with the molecule being attached to only one of the passivated areas, but not to the other. In contrast, those simulations where $L$ is only slightly larger than $d$ most likely end up with the formation of the junction. To this end, both the molecule and the tips bend and distort slightly to make room for the chemical bond. Typical final configurations are shown in Figs. 1-3. For armchair wedges, the molecule is bound to carbon atoms at the side of the wedge tip and is oriented roughly perpendicular to the graphene planes. This effect is due to the particular directionality of the carbon-sulfur covalent bond, which gives nontrivial structural configurations. We have found a similar effect for the mixed wedges. In contrast, zigzag wedges are terminated in a single carbon atom. In this case, BDT molecules find it easier to orient roughly parallel to the graphene layers.

We have addressed the mechanical stability of the junctions at room temperature by carrying out first-principles moleculardynamics simulations using a Nose thermostat with target temperature of $300 \mathrm{~K}$. Typical simulations have run for a few hundred steps, with step durations of $1 \mathrm{fs}$. We have found that the molecule and carbon atoms at the junction area undergo collective motions as the vibrational modes of the junction are activated. But even with these strong vibrations, the integrity of the junction is maintained throughout these short simulations. This short-time robustness suggests that these junctions are stable at room temperature.

We analyze now the electronic structure of the three wedge junction types shown in Figs. 1-3. The top panels in Fig. 4 show the total density of states (DOS) of the junctions without bridging molecules, which suggests a tentative idea of how the lead's conduction channels are distributed in energy. Note that the DOS shape of the wedges [Fig. 4 (a1-b1-c1)] resembles closely bulk graphene's DOS, as is the case of large-width graphene nanoribbons. ${ }^{45}$ Armchair wedge junctions show a small gap about the Fermi energy $E_{F}$; see Fig. 4 (a1). Mixed and zigzag wedges show in contrast additional peaks about $E_{F}$ associated with edge states. These edge states hybridize with BDT's frontier orbitals giving rise to a finite DOS projected on the molecule orbitals (PDOS) as shown in Fig. 4 (b3-c3).

We find that zigzag wedges are spin-polarized. The nature and features of this spin polarization are similar to that found in zigzag nanoribbons ${ }^{30,31}$ and zigzag nanoislands. ${ }^{33}$ Zigzag nanoislands show a finite spin polarization in agreement with Lieb's theorem for bipartite lattices, ${ }^{33}$ which states that the total spin $S$ of the exact ground state of the Hubbard model is equal to half the lattice imbalance $N_{z}=N_{A}-N_{B}$, where $N_{A, B}$ are the total number of sites in sublattices $A$ and $B$, respectively. ${ }^{46}$ Atoms in the $A(B)$ nanoisland sublattices are up- (down-) spin-polarized, resulting in an uncompensated ferrimagnetic spin arrangement. In our case, the wedges shown in Fig. 3 have three more atoms in the $A$ sublattice and therefore the lattice imbalance is $N_{z}=3$. By

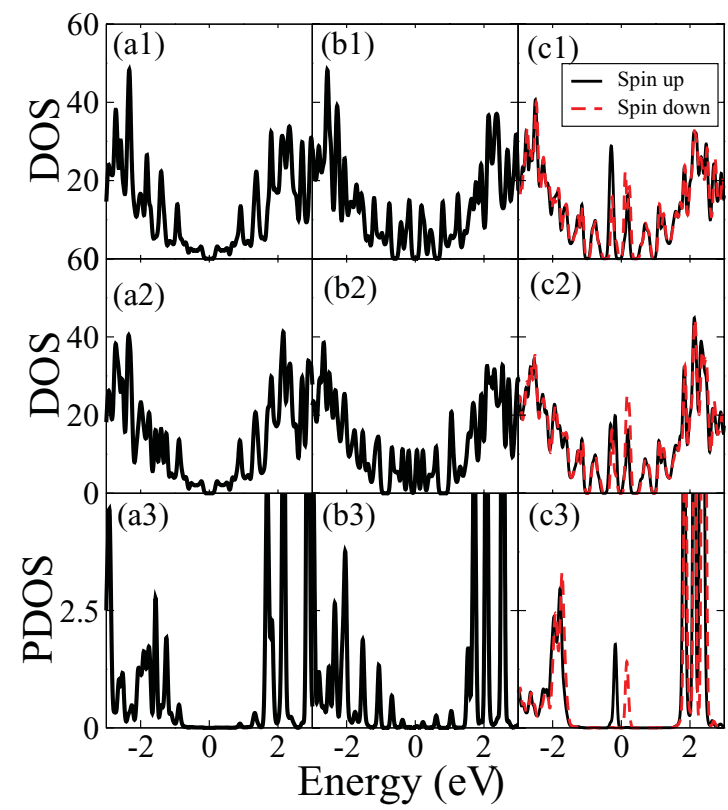

FIG. 4. (Color online) (a1-b1-c1) Total DOS (in arbitrary units) of graphene wedge junctions without a bridging molecule; (a2b2-c2) same, but with a bridging BDT molecule. (a3-b3-c3) DOS (in arbitrary units) of the BDT-bridged junctions, projected onto the molecule orbitals. Left, center, and right panels correspond to armchair, mixed, and zigzag wedges. Wedge-wedge distances correspond to the shortest-distance cases shown in Figs. 1-3. Solid black and dashed red lines in panels (c1), (c2), and (c3) correspond to up and down spins.

inspecting the Mulliken populations, we have found that the spin polarization of each sublattice is maximal at the atoms in the wedge tips, and decays slowly toward the inner side of the graphene sheets. As a consequence, to compute the spin polarization of each sublattice, one must take into account all the carbon atoms in the sheets (and also the hydrogen atoms at the edges). By doing so, we have found a total magnetic moment $m=2 S$ of 2.8, using both LDA and GGA functionals. This result is in good agreement with Lieb's theorem, given the approximate character of the Mulliken method for the computation of electronic populations. This spin polarization is also transmitted to the BDT molecule, as demonstrated by the peaks in the PDOS shown in Fig. 4 (c3). In contrast, armchair and mixed wedges are nonmagnetic, again in agreement with Lieb's theorem.

To visualize better how these edge states are distributed spatially, we have plotted the wedge local density of states (LDOS), integrated in a window from -0.5 to $+0.5 \mathrm{eV}$ about the Fermi energy. We have found that charge is driven away from the edges for armchair wedges, as is shown in Fig. 5(a). In contrast, mixed wedges have large edge segments with zigzag arrangements, which show accumulation of charge. The tip of these wedges, however, shows an armchair arrangement; consequently, charge is driven away from the wedge tip, as is shown in Fig. 5(b). Zigzag wedges show spin-polarized edge states all the way up to the wedge tip, except for the last carbon atom, as can be seen in the LDOS plotted in Fig. 5(c).

The junctions transmission coefficients $T(E)$ are shown in the three bottom panels in Fig. 6. The top panels show 


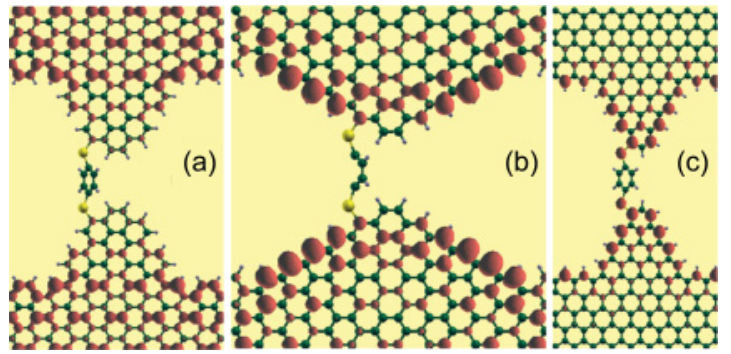

FIG. 5. (Color online) Local density of states integrated in an energy window around the Fermi energy for (a) armchair, (b) mixed, and (c) zigzag wedges. For zigzag wedges we show only the majority spin component of the charge.

the transmission coefficients multiplied by $10^{7}$ for similar junctions, where the BDT bridging molecules have been replaced by passivating hydrogen atoms. In addition, the gap length in this second set of junctions has been adjusted a little to allow for a tiny but finite overlap between those terminating hydrogen atoms, so that the transmission coefficient would be nonzero. Notice that the behavior of the top panels roughly matches that of the equivalent bottom panels, signaling that the functionalities of these devices are brought about by the specific wedge shape in each case. This was of course expected given the known nonfunctional behavior of BDT molecules when contacted by noble metal electrodes. The transmission of armchair wedges is quite featureless and small, while for mixed wedges the transmission is small but shows a marked dip pinned at the Fermi energy. Zigzag wedges are much more interesting. Here, sharp transmission peaks around the Fermi energy are apparent. $T$ shows only one spin-polarized peak on each side of the Fermi level which is correlated with the peaks in the DOS curves shown in the right panels in Fig. 4. We expect that the sharp peaks should give rise to much higher values of the current in the $I-V$ characteristics than for the former two types of junctions. Furthermore, spin-filtering phenomena should appear.

We calculate the current by integrating the bias-dependent transmission coefficients $T(E, V)$ in an energy window given

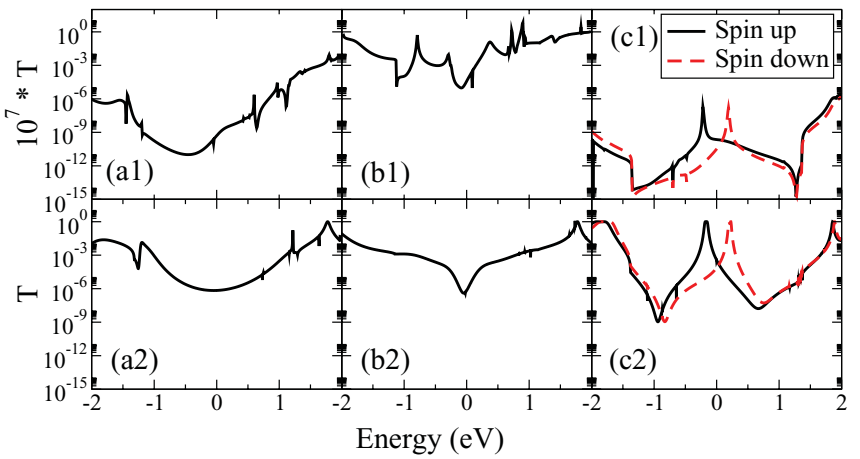

FIG. 6. (Color online) (a1-b1-c1) Transmission coefficients of graphene wedge junctions without a bridging molecule multiplied by $10^{7}$; (a2-b2-c2) same, but with a bridging BDT molecule. Left, center and right panels correspond to armchair, mixed, and zigzag wedges. Wedge-wedge distances correspond to the shortest-distance cases shown in Figs. 1-3. Solid black and dashed red lines in panels (c1) and (c2) correspond to up and down spins.
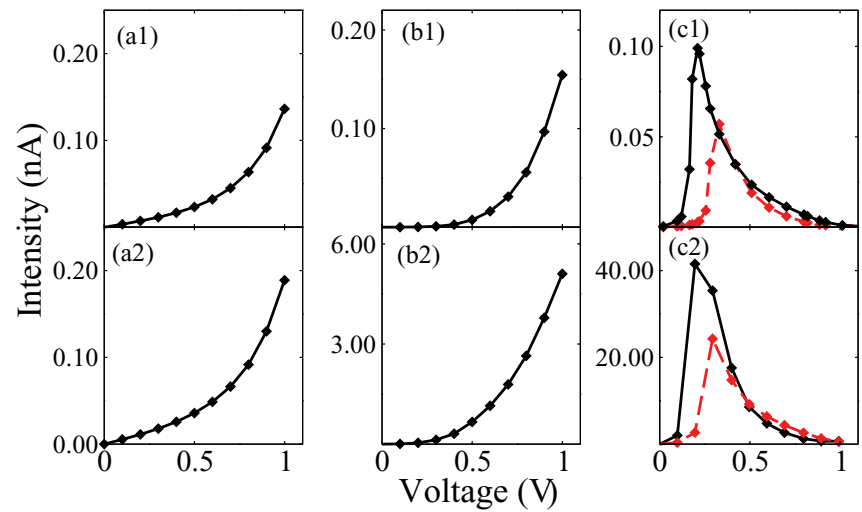

FIG. 7. (Color online) $I-V$ characteristics of the system composed of wedge-BDT-wedge junctions for (a) armchair, (b) mixed, and (c) zigzag edges. Top/bottom panels correspond to longest/shortest distances between the graphene edges shown in Figs. 1-3. Solid black and dashed red lines in panels (c1) and (c2) correspond to up and down spins.

by the voltage bias. The $I-V$ characteristics for armchair junctions show Ohmic-like behavior at small voltages as shown in Figs. 7 (a1-a2). The absence of transmission resonances close to the Fermi level is manifested in the small values of the current. This behavior can also be understood by inspecting the LDOS shown in Fig. 5(a), where it is apparent that the charge within the graphene wedges accumulates far away from the junction. Mixed junctions show a semiconducting shape, where a gap is apparent followed by a steep rise in the conductance. Further, the current changes by more than one order of magnitude when approaching the two edges from 9.5 to $7.2 \AA$. This can again be understood by inspecting Fig. 5(b), where it is clear that the BDT molecule attaches to atoms having a finite LDOS for the closer distance [corresponding to Fig. 2(b)], but not when the edges are separated by $9.5 \AA$, which corresponds to Fig. 2(a). For zigzag junctions, the sharp spin-polarized transmission peaks at low energies result in a spin-filtering effect which we show in Fig. 7 (c1-c2). These $I-V$ characteristics also show a strong NDR effect, whose origin can be traced to a shift in energy and a change in shape of the spin-polarized transmission peaks shown in Fig. 6 (c1-c2) as the bias voltage increases, as we will show below. Reducing the distance between the two edges from 9.6 to $8.2 \AA$ yields a change in the current of more than two orders of magnitude. To understand this change, one must again look at Fig. 4(c) and realize that when the molecule attaches right at the wedge tip [Fig. 3(a)], there is no charge available for transport at low voltages, while if the molecule attaches to the neighboring carbon atom [Fig. 3(b)], then the edge state hybridizes strongly with the sulfur atom, as is shown in Fig. 5(c).

To understand better the origin of the NDR effect in zigzag BDT junctions, we plot in Fig. 8 the transmission coefficients $T(E, V)$ as a function of energy $E$ for several voltages $V$ increasing from 0 to $0.6 \mathrm{~V}$. Notice that the height of the peaks decreases and splits in two as the voltage is increased. To understand this behavior, we focus now on the molecular orbitals associated with the transmission peaks. These are localized mainly at the sulfur atoms placed at the two edges of the molecule. They are responsible for the 


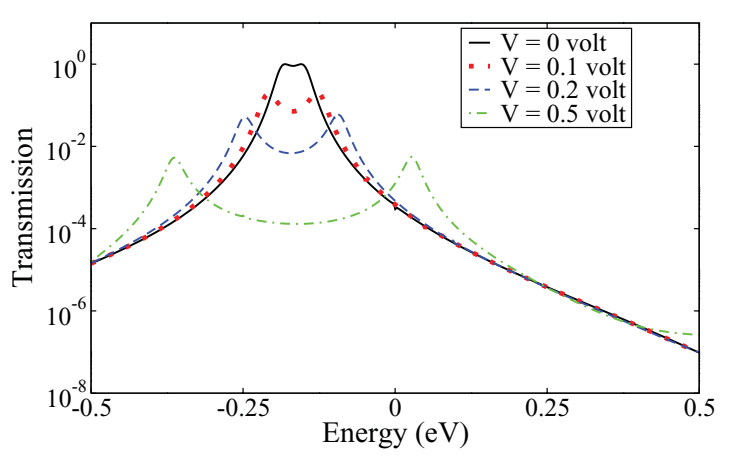

FIG. 8. (Color online) Spin-polarized energy-dependent transmission of a BDT zigzag junction, plotted for voltages increasing from 0 to $0.6 \mathrm{~V}$ in a logarithmic scale.

coupling between molecule and electrodes. These orbitals give rise to bonding and antibonding molecular orbitals across the molecule. It turns out that the impact of a voltage bias on these frontier molecular orbitals is strong and nontrivial. When $V$ increases the degree of localization at the coupling atoms, they become asymmetrically coupled to the electrodes and their energy moves in opposite energy directions. The evolution of the bonding and antibonding states produced by the above molecular orbitals with the bias voltage is the main mechanism triggering the NDR behavior in these junctions. The mechanism, however, is generic and could be applied to any other junction where frontier molecular orbitals are located close to the Fermi energy. We therefore suggest that a necessary condition for the appearance of NDR in small molecules coupled to metallic electrodes is the existence of these side molecular orbitals. A simple model that captures the essential features of the NDR effect consists of two metallic monatomic chains having localized states at the edges which interact across a vacuum region, as we explain in the Appendix. The more localized the states and the less interaction between them, the lower the voltage where the NDR peak appears and the higher the value of the peak to valley ratio but the lower also the value of the current. The key features of an efficient NDR device are a low value of the peak voltage and a high peak-to-valley value of the current. These can be tuned by balancing the degree of localization of the edge states and their coupling across the vacuum region.

\section{WEDGE-BPD-WEDGE JUNCTIONS}

To test for the impact of the contact atoms on the transport properties, we have carried out simulations where the bridging BDT molecule in the junctions shown in Figs. 1(a), 2(a), and 3(a) has been replaced by a BPD molecule. This means that the new set of calculations has been performed only for intermediate junction gap lengths of about $9 \AA$, and with the geometries shown in Fig. 9. The total DOS and the DOS projected onto BPD's nitrogen and carbon atoms are shown in Figs. 10 (a1-b1-c1) and 10 (a2-b2-c2), respectively. Notice that the DOS shows now a sharp peak close to the Fermi level for the three wedge junctions. To check for the spatial location of the state associated with that peak, we have plotted in Fig. 9 the LDOS integrated in a small window in energy which only retains the peak. The figure demonstrates that the

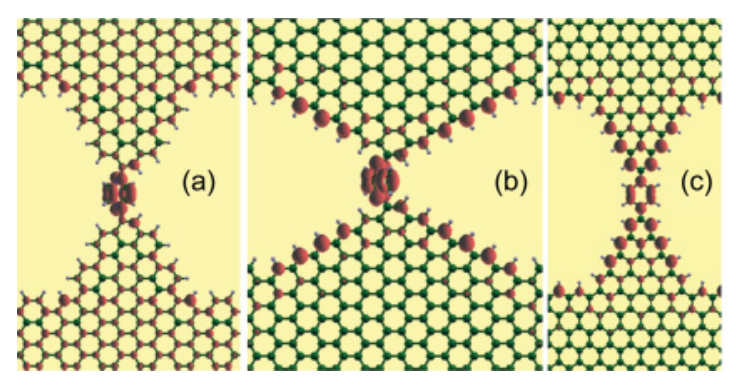

FIG. 9. (Color online) Local density of states of BPD junctions integrated in an energy window around the Fermi energy for (a) armchair, (b) mixed, and (c) zigzag wedges. For zigzag wedges, we show only the majority spin component of the charge.

state corresponds to a molecular orbital extended throughout the whole molecule now. The corresponding transmission peak or Fano-like resonances, shown in the bottom panels in Fig. 10, are pinned at the Fermi energy and should lead to a dramatic increase in the current of armchair and mixed wedge junctions, as compared to their equivalent BDT junctions. The $I-V$ curves of these wedge junctions are shown in Fig. 11. Notice indeed that the current of the armchair and mixed wedge junctions increases by several orders of magnitude, although both $I-V$ curves retain the Ohmic- and semiconducting-like behaviors displayed in BDT junctions. BPD armchair and mixed junctions do not show NDR behavior because the new molecular state associated with the PDOS peak is localized inside the molecule and not at its edges, and is therefore not strongly affected by the bias voltage. Zigzag BPD junctions also show an increase of the current of two orders of magnitude compared to Fig. 7 (c1) although the effect is not as dramatic as for armchair and mixed junctions; see Fig. 11(c). To understand this, we compare in Figs. 9(a)-9(c) the amount of charge associated with the molecular state, which is clearly smaller for the zigzag junction. The SP and NDR behaviors of the zigzag BPD junction shown in Fig. 11(c) are not as marked as for the BDT molecule as well. The reason behind the reduction of the two effects can also be traced to the new molecular state responsible for the current increase. The point is that this

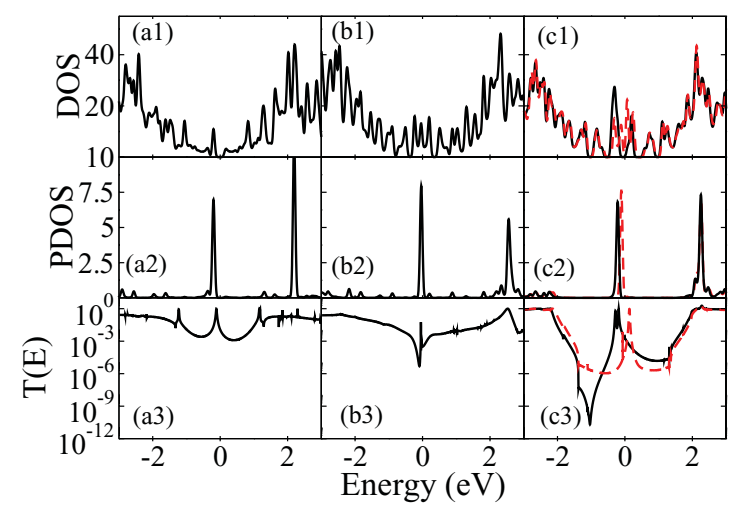

FIG. 10. (Color online) (a1-b1-c1) Total DOS. (a2-b2-c2) DOS projected on the molecule atoms. (a3-b3-c3) Transmission coefficients of wedge-BPD-wedge junctions. Left, middle, and right panels correspond to armchair, mixed, and zigzag wedges. Solid black and dashed red lines in panels (c1), (c2), and (c3) correspond to up and down spins. 

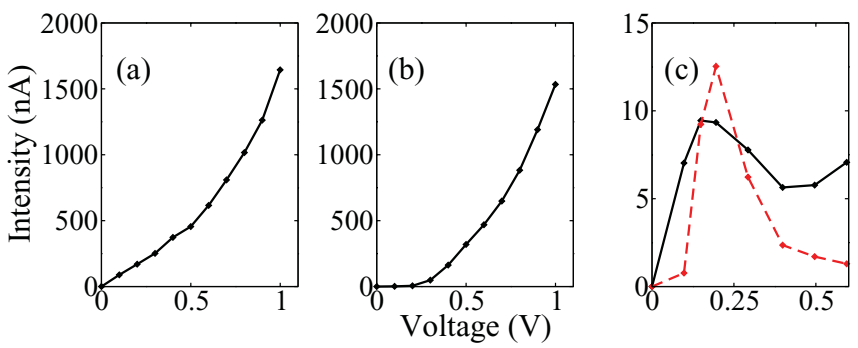

FIG. 11. (Color online) Current as a function of bias voltage for BPD junctions with (a) armchair, (b) mixed, and (c) zigzag wedges.

molecular orbital is not as affected by the graphene edge states as the sulfur atoms in BDT junctions were.

Figure 12 shows the behavior of the energy-dependent transmission coefficient of a BPD mixed junction as the voltage is ramped up from $0 \mathrm{~V}$. Notice how a Fano-like resonance develops such that a dip feature emerges as $V$ increases. Further, notice that the peak and the dip move in opposite energy directions to make the resonance wider. Because the peak moves to lower negative energy values when $V$ increases, a large fraction of its weight remains out of the integration window. This is the reason why the $I-V$ characteristics of mixed junctions exhibit a semiconducting shape. The voltage dependence of the transmission coefficients of zigzag junctions is shown in Fig. 13. Here the split frontier orbitals are nondegenerate even at zero voltage. Ramping up the bias further splits the peaks. However, the position and height of the dominant peak do not change much. This peak corresponds to the molecular orbital centered within the BPD molecules, which is not too strongly affected by the bias. In contrast, the position and height of the other peak are severely affected by the voltage. This NDR effect is not as marked as for the zigzag BDT junction because here it results in a tradeoff of the evolution of the different transmission peaks.

\section{CONCLUSIONS}

This article presents an analysis of the anchoring of benzene-dithiolate and bipyridine molecules to a variety of graphene wedge electrodes. It also presents results of the electronic and transport properties of the ensuing singlemolecule junctions. The article shows how the diverse electronic structure of the graphene edges is transmitted into

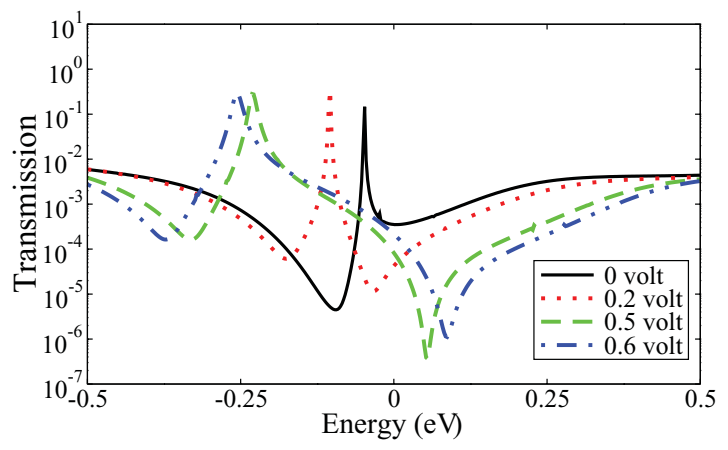

FIG. 12. (Color online) Energy dependence of the transmission coefficient of a BPD mixed junction for voltage biases ramped from 0 to $0.6 \mathrm{~V}$.

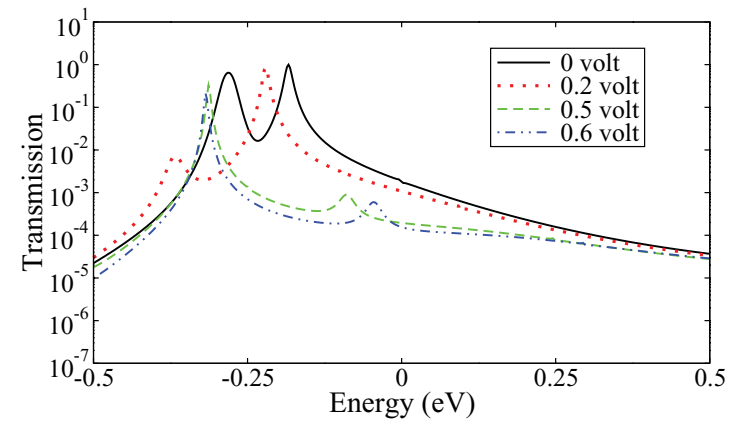

FIG. 13. (Color online) Energy dependence of the spin-up transmission coefficient of a BPD zigzag junction for voltage biases ramped from 0 to $0.6 \mathrm{~V}$.

a rich variety of transport phenomena, which include spinpolarized transport, negative differential resistance behavior, and Fano-like resonances. On the positive side, the wealth of results obtained for simple nonfunctional molecules indicates that graphene-wedge single molecule electronics could be exploited as a fruitful playground for new functionalities provided that the shape of the edges could be controlled. On the negative side, such a variety of behaviors should lead to strong tribological effects and large variability in the electrical response of these junctions if edge shape morphology is not controlled at the atomic scale.

\section{ACKNOWLEDGMENTS}

This work was supported by the the Spanish Ministry of Education and Science (project FIS2009-07081) and the Marie Curie network NanoCTM. V.M.G.S. thanks the Spanish Ministerio de Ciencia e Innovación for a Ramón y Cajal fellowship (RYC-2010-06053). J.F. acknowledges discussions with C. J. Lambert, M. Calame, F. Prins, and R. Fasel.

\section{APPENDIX: SIMPLE MODEL FOR THE NDR EFFECT IN GRAPHENE-WEDGE JUNCTIONS}

The main mechanism behind the NDR effect in molecules between graphene sheets and in many other molecular junctions is produced by the localization of states at the contacts which are weakly coupled through the molecular backbone and have energies close to the Fermi level. These states separate as the bias increases, which decreases the transmission through the junction near the Fermi level and reduces the current for biases larger than a certain bias (threshold voltage). As a consequence, the increase of the integration voltage window cannot compensate for the reduction of the transmission, and the total current is reduced from the maximum obtained at lower absolute voltages.

A simple picture that can grasp this effect can be elaborated with a two-level system such as the one shown in Fig. 14. Both levels are located at the contacts and have an energy equal to $\varepsilon_{0}$, which in this case is a bit below the Fermi level of the leads, $E_{F}$, but could also be a bit above. The coupling between the levels, $\gamma$, greatly influences the shape of the transmission. If $\gamma$ is big enough, both levels interact strongly and give rise to bonding and antibonding states separated by $2 \gamma$. Since the system is symmetric, these levels produce Breit-Wigner 


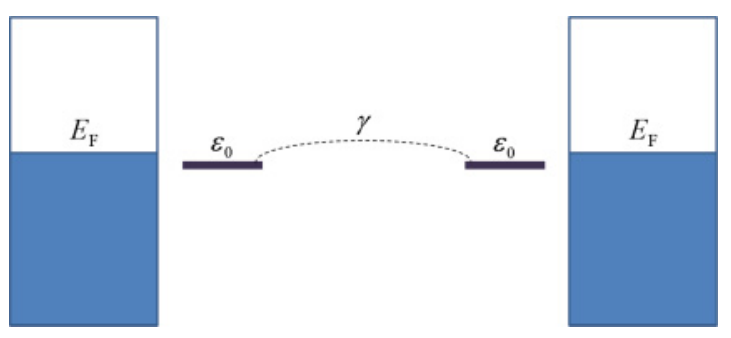

FIG. 14. (Color online) Two-level system used to simulate NDR mechanisms produced by the localization of states near the contacts.

resonances of height equal to 1 (or an integer multiple of 1 in the case of degenerate states). When $\gamma$ decreases, both levels merge and the bonding-antibonding system transforms into a system with two asymmetric levels located at the same energy. As a consequence, the Breit-Wigner resonances merge into a single resonance which corresponds to the transmission of two asymmetrically coupled levels and whose width decreases as $\gamma$ is further reduced. An example of a system with contact states relatively well coupled through the molecule is the benzenedithiolate molecule between gold leads, whose transmission shows a broad HOMO resonance of height almost equal to 1 produced by states located on the sulfurs. An example of system with contact states weakly coupled through the molecule is, e.g., any alkane-dithiolate molecule between gold leads, whose sulfur states do not interact across the molecular backbone and give rise to transmission resonances of heights much smaller than 1 .

It is important that such levels are relatively near the Fermi level, localized on the contacts, and coupled through the molecule, so that they produce sharp resonances. Under such conditions, the effect of the bias is enhanced due to the quick misalignment of the resonances, which strongly reduces the transmission and the current and gives rise to clear NDR effects. The sharper the resonance and the closer it is to the Fermi level, the better the NDR parameters. However, if the levels strongly hybridize with the electrodes, the transmission features are much broader and the NDR develops at much higher voltages or does not develop at all. On the other hand, if sharp resonances are located away from the Fermi level, the resulting NDR peak broadens and moves to higher voltages since the effect of the reduction of the height of the resonances is smaller away from the position of the resonance peak. Something similar happens if the resonances are close to the Fermi level but are broad instead. In that case, the height of the transmission features decreases more slowly and the NDR fades again.

It is possible to conclude then that in all cases with localized states on the coupling region, the development and shape of the NDR depend on the localization of such states and their interaction across the molecule. This last variable can be simplified and the system can be transformed into a simple case of two coupling atoms with a vacuum region in between, which would mimic an ideal low-conductive molecule. This model has basically two variables: the distance between the coupling atoms and the electrodes and the length of the vacuum region between them. The first variable determines the localization of the states, which is directly related to the width of the transmission resonances, and the second variable determines the height and shape of the resonances (i.e., resonances of height equal to 1 when the distance is small and the atoms are well coupled, and resonances of smaller height when the distance is large and the atoms are weakly coupled). A third variable could be the position of the contact states, determined by the type of atom.

We simulated this system with various types of light elements typically used to couple molecules to metallic electrodes ( $\mathrm{C}, \mathrm{N}, \mathrm{O}$, and $\mathrm{S}$ ) bonded to perfect atomic chains made of carbon (1.28 $\AA$ of separation between atoms), which would mimic perfect metallic electrodes. We chose an initial distance of $3.70 \AA$ A between the coupling atoms, which couples them but not very strongly. We used the LDA approximation for the exchange and correlation potential, a real space mesh cutoff of $200 \mathrm{Ry}$, and a double-zeta polarized (DZP) basis set. Even though some of the cases would be magnetic, we did not include the possibility of spin polarization to further simplify the system and focus only on the NDR effect. We relaxed the position of the coupling atoms until the forces were smaller than $0.05 \mathrm{eV} / \AA$, which gave distances to the first atom of the carbon chain of $1.31,1.19,1.17$, and $1.57 \AA$ for $\mathrm{C}, \mathrm{N}, \mathrm{O}$, and $\mathrm{S}$, respectively.

The results obtained with the initial parameters are shown in Fig. 15. As can be seen, all currents are almost Ohmic and do not show any sign of NDR. This is because at the relaxed distances all coupling atoms hybridize strongly with the carbon chain and do not produce resonances in the transmission coefficients but plateaus of almost constant transmission, like the one shown in Fig. 20(a). This case, however, is not typical in molecular electronics systems, where the coupling to the electrodes is not as strong and the states on the coupling atoms are more localized (unless the molecules are very small and diatomic-like ${ }^{47}$ ). To further increase the localization, we artificially separate the coupling atom $0.5 \AA$ from the carbon chain. This change produces the results shown in Fig. 16. A clear NDR behavior appears now in all cases. The localization produces transmissions that have a resonance-like shape. The
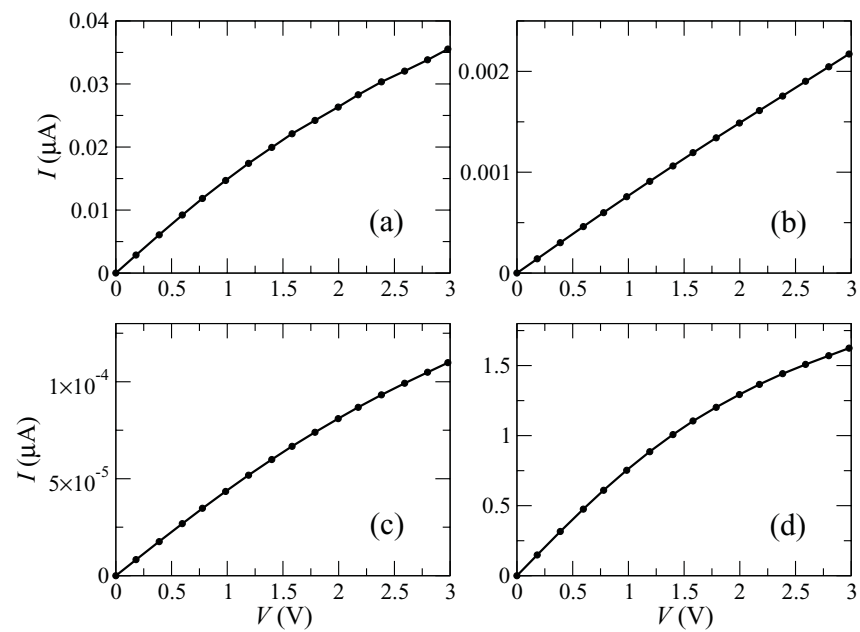

FIG. 15. Current as a function of voltage for junctions made of carbon (a), nitrogen (b), oxygen (c), and sulfur (d) atoms connected to perfect atomic carbon chains and separated by a vacuum region of $3.70 \AA$. The atomic coordinates of the contact atoms have been relaxed. 

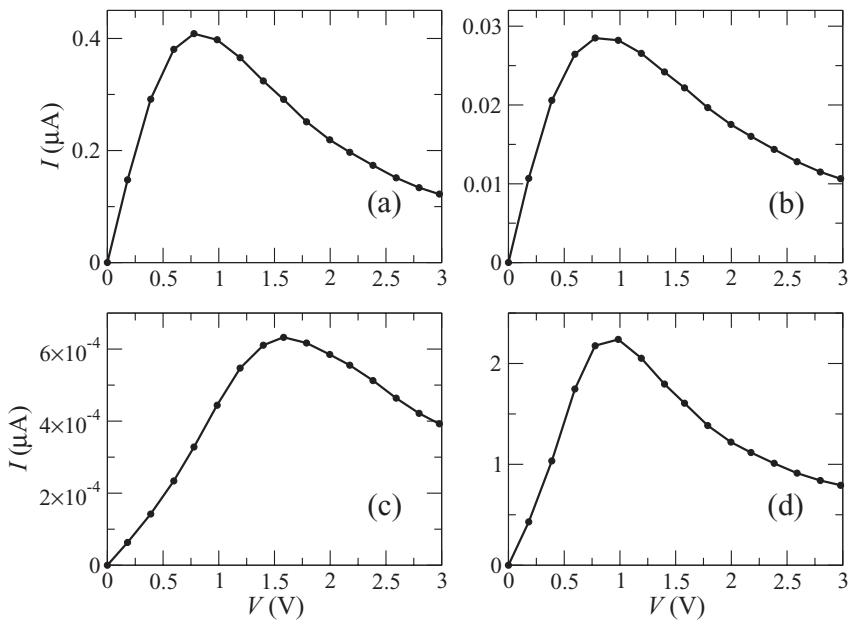

FIG. 16. Current as a function of voltage for junctions made of carbon (a), nitrogen (b), oxygen (c), and sulfur (d) atoms connected to perfect atomic carbon chains and separated by a vacuum region of $3.70 \AA$. The distance between the atoms and the chain has been artificially increased $0.50 \AA$ from the relaxed distance.

height of such resonances is reduced and the peak is divided in two smaller peaks (corresponding to the coupling states on each side) as the voltage increases.

When the coupling atom is carbon, the atomic states closer to the Fermi level, which coincide with the main transmission peaks, are located a bit above $E_{F}$. In the case of $\mathrm{N}$ and $\mathrm{O}$, they appear at lower energies (very close to the Fermi level and below it, respectively), following the expected evolution due to the increasing nuclear attraction from $\mathrm{C}$ to $\mathrm{O}$. In the case of $\mathrm{S}$, the states are a bit above those of $\mathrm{O}$, which is also expected due to the lower electronegativity of $\mathrm{S}$ compared to O. Notice also that the current is highest when the coupling atom is $\mathrm{S}$ and decreases from $\mathrm{C}$ to $\mathrm{O}$. This is due to the nuclear attraction again, which increases the atomic localization of the states and therefore decreases their interaction across the
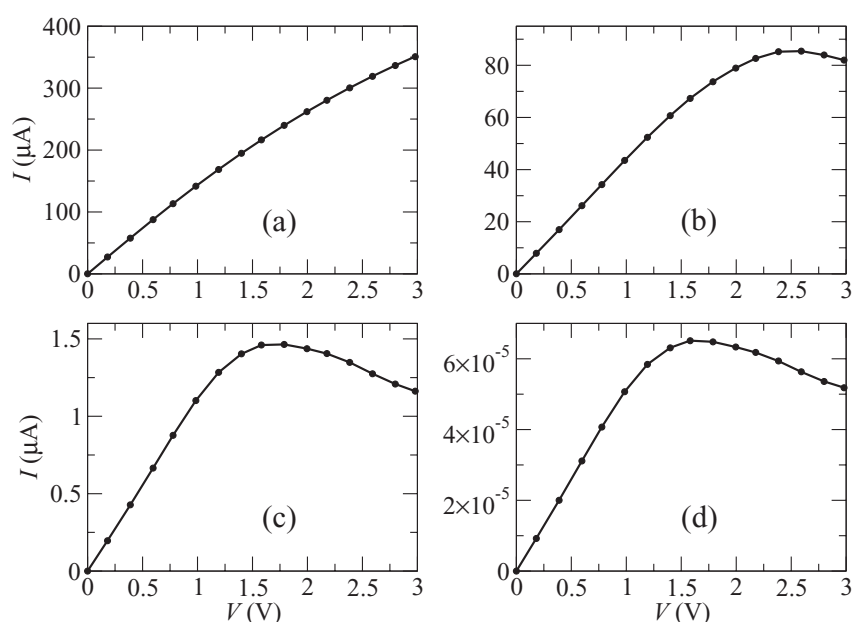

FIG. 17. Current as a function of voltage for junctions made of sulfur atoms connected to perfect atomic carbon chains, a bit stretched from the relaxed distance and separated by a vacuum region of $1.74 \AA$ (a), $2.74 \AA$ (b), $3.74 \AA$ (c), and $5.04 \AA$ (d).
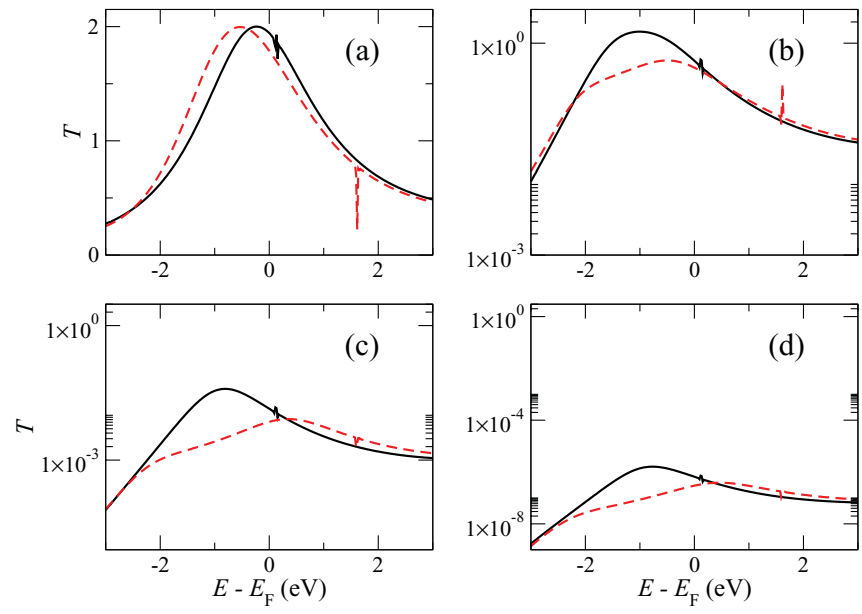

FIG. 18. (Color online) Transmission of junctions made of sulfur atoms connected to perfect atomic carbon chains, a bit stretched from the relaxed distance and separated by a vacuum region of $1.74 \AA$ (a), $2.74 \AA$ (b), $3.74 \AA$ (c), and $5.04 \AA$ (d). Continuous and dashed lines correspond to the transmission at 0 and $3 \mathrm{~V}$, respectively. Notice that in panel (a) the scale is linear.

vacuum gap as the atomic number increases along the same row of the Periodic Table or decreases along the same column.

The element that gives the larger coupling across the vacuum gap is sulfur. We use then this element to study the effect of changing the distance between the contact atoms, maintaining the distance between $\mathrm{S}$ and the carbon chain equal to the relaxed distance plus $0.3 \AA$ to produce some localization. The results are shown in Fig. 17. As can be seen, the $I-V$ characteristics evolve from almost Ohmic to NDR-like. This evolution can be explained by taking into account the transmission, shown in Fig. 18. When the atoms are very close, the transmission around the Fermi level has a Breit-Wigner resonance of height equal to 2 produced by two degenerate states corresponding to the $p$ orbitals perpendicular
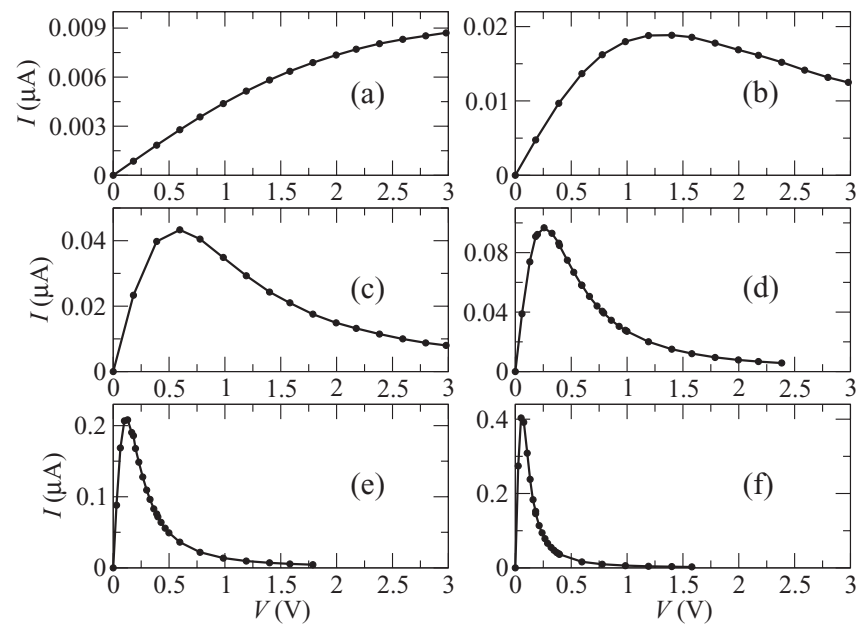

FIG. 19. Current as a function of voltage for junctions made of nitrogen atoms connected to perfect atomic carbon chains, separated by a vacuum region of $3.70 \AA$ and stretched from the relaxed distances $0.20 \AA$ (a), $0.40 \AA$ (b), $0.60 \AA$ (c), $0.80 \AA$ (d), $1.00 \AA$ (e), and $1.20 \AA$ (f). 

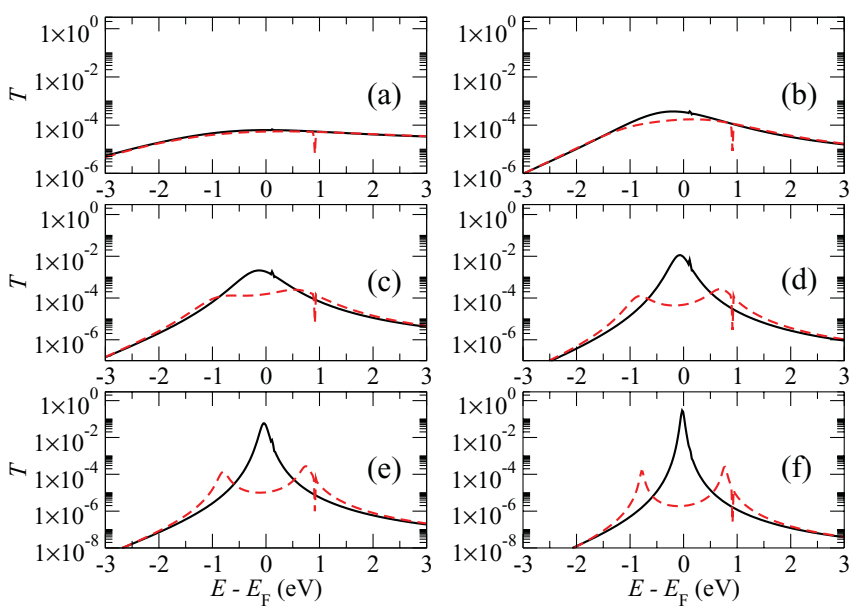

FIG. 20. (Color online) Transmission of junctions made of nitrogen atoms connected to perfect atomic carbon chains, separated by a vacuum region of $3.70 \AA$ and stretched from the relaxed distances $0.20 \AA$ (a), $0.40 \AA$ (b), $0.60 \AA$ (c), $0.80 \AA$ (d), $1.00 \AA$ (e), and $1.20 \AA$ (f). Continuous and dashed lines correspond to the transmission at 0 and $1.6 \mathrm{~V}$, respectively.

to the transport direction, which couple weakly to the same orbitals of the other sulphur atom across the gap. In such a situation, the delocalized molecular states screen the effect of the bias voltage and the resonance moves only slightly to lower energies due to charge transfer. ${ }^{39}$ If the distance increases, however, both states uncouple and separate. This allows them to follow the chemical potential of each electrode at finite biases. In such a case, the net effect on the transmission produced by the bias is a drastic reduction of the resonance height and its further separation in two satellites.

The factor that dramatically improves the quality of NDR is the localization of the coupling states, which can be increased by varying the distance between the coupling atom and the carbon chain: the larger the distance, the more localized the atomic states and vice versa. We used $\mathrm{N}$ to study this effect, since it has the closest states to the Fermi level and can produce the clearest NDR signals. The results are shown in Fig. 19. As can be seen, the height of the NDR peak increases and the voltage at which it appears decreases as a function of distance. This evolution can be easily understood by looking at the transmission as a function of bias, shown in Fig. 20 at 0 and $1.6 \mathrm{~V}$. When the distance is small, the large hybridization with the carbon chain does not produce any resonance and therefore the transmission does not change with bias. As the distance increases, a transmission peak starts to develop. The larger the distance, the higher and sharper the peak and the bigger the effect of the bias voltage, which splits the peak in two smaller satellites. This can be explained by taking into account that at very large distances, the coupling between the contact atoms is larger than the coupling to the chain; this coupling effectively produces molecular states in the middle of the junction that generate very sharp resonances in the transmission. When the voltage increases, the states of each atom follow the chemical potential of their electrode and, if the screening is not very big, the system transforms into a system of two asymmetric states, each of which produces a smaller transmission peak.

In summary, a very simple model that takes into account only the coupling of the contact atoms to the electrodes and the coupling between them can be used to describe fairly well a NDR mechanism that is expected to appear in many molecular junctions. In reality, the molecular backbone would change the hybridization and position of the contact states but the conclusions reached here would still be valid, i.e., the more localized and closer to the Fermi level the contact states are, the better is the NDR.

Note added in proof. After completion of this work, R. Fasel brought our attention to Ref. 48, where the magentism of zigzag wedges is also discussed.
${ }^{1}$ L. A. Ponomarenko, F. Schedin, M. I. Katsnelson, R. Yang, E. W. Hill, K. S. Novoselov, and A. K. Geim, Science 320, 356 (2008).

${ }^{2}$ M. H. Gass, U. Bangert, A. L. Bleloch, P. Wang, R. R. Nair, and A. K. Geim, Nat. Nanotech. 3, 676 (2008).

${ }^{3}$ D. V. Kosynkin, A. L. Higginbotham, A. Sinitskii, J. R. Lomeda, A. Dimiev, B. K. Price, and J. M. Tour, Nature (London) 458, 872 (2009).

${ }^{4}$ L. Liying Jiao, L. Zhang, X. Wang, G. Diankov, and H. Dai, Nature (London) 458, 877 (2009).

${ }^{5}$ H. Wang, D. Nezich, J. Kong, and T. Palacios, Electron Device Lett. 30, 547 (2009).

${ }^{6}$ F. Schedin, A. K. Geim, D. V. Morozov, E. W. Hill, P. Blake, M. I. Katsnelson, and K. S. Novoselov, Nat. Mater. 6, 652 (2007).

${ }^{7}$ A. V. Rozhkov, G. Giavaras, Y. P. Bliokh, V. Freilikher, and F. Nori, Phys. Rep. 503, 77 (2011).

${ }^{8}$ J. M. Tour, Molecular Electronics (World Scientific, Singapore, 2005).

${ }^{9}$ L. Bogani and W. Wernsdorfer, Nat. Mater. 7, 179 (2008).
${ }^{10}$ J. Ferrer and V. M. García-Suárez, J. Mater. Chem. 19, 1696 (2009).

${ }^{11}$ X. Jia1, M. Hofmann, V. Meunier, B. Sumpter, J. CamposDelgado, J. M. Romo-Herrera, H. Son, Y-P. Hsieh, A. Reina, J. Kong, M. Terrones, and M. S. Dresselhaus, Science 323, 1701 (2009).

${ }^{12}$ F. Prins, A. Barreiro, J. W. ruitenberg, J. S. Seldenthuis, N. AliagaAlcalde, L. M. K. Vandersypen, and H. S. J. van der Zant, Nano Lett. 11, 4607 (2011).

${ }^{13}$ Y. D. He, H. L. Dong, T. Li, C. L. Wang, W. Shao, Y. J. Zhang, L. Jiang, and W. P. Hu, Appl. Phys. Lett. 97, 133301 (2010).

${ }^{14}$ J. Cai, P. Ruffieux, R. Jaafar, M. Bieri, T. Braun, S. Blankenburg, M. Muoth, A. P. Seitsonen, M. Saleh, X. Feng, K. Müllen, and R. Fasel, Nature (London) 466, 470 (2010).

${ }^{15}$ M. A. Reed, C. Zhou, C. J. Muller, T. P. Burgin, and J. M. Tour, Science 278, 252 (1997).

${ }^{16}$ M. Di Ventra, S. T. Pantelides, and N. D. Lang, Phys. Rev. Lett. 84, 979 (2000). 
${ }^{17}$ K. Stokbro, J. Taylor, M. Brandbyge, J. L. Mozos, and P. Ordejón, Comput. Mater. Sci. 27, 151 (2003).

${ }^{18}$ Y. Xue and M. A. Ratner, Phys. Rev. B 68, 115406 (2003); 68, 115407 (2003).

${ }^{19}$ J. Campos-Delgado, I. O. Maciel, D. A. Cullen, D. J. Smith, A. Jorio, M. A. Pimenta, H. Terrones, and M. Terrones, ACS Nano 4, 1696 (2010).

${ }^{20}$ J. M. Romo-Herrera, D. A. Cullen, E. Cruz-Silva, D. Ramírez, B. G. Sumpter, V. Meunier, H. Terrones, D. J. Smith, and M. Terrones, Adv. Funct. Mater. 19, 1193 (2009).

${ }^{21}$ J. Chen, M. A. Reed, A. M. Rawlett, and J. M. Tour, Science 286, 1550 (1999).

${ }^{22}$ S. Barraza-López, K. Park, V. M. García-Suárez, and J. Ferrer, J. Appl. Phys. 105, 07E309 (2009).

${ }^{23}$ S. Barraza-López, K. Park, V. M. García-Suárez, and J. Ferrer, Phys. Rev. Lett. 102, 246801 (2009).

${ }^{24}$ C. M. Finch, V. M. García-Suárez, and C. J. Lambert, Phys. Rev. B 79, 033405 (2009).

${ }^{25}$ D. Dragoman and M. Dragoman, Appl. Phys. Lett. 90, 143111 (2007).

${ }^{26}$ H. Cheraghchi and K. Esfarjani, Phys. Rev. B 78, 085123 (2008).

${ }^{27}$ L. A. Agapito and H. P. Cheng, J. Phys. Chem. C 111, 14266 (2007).

${ }^{28}$ M. Koleini, M. Paulsson, and M. Brandbyge, Phys. Rev. Lett. 98, 197202 (2007)

${ }^{29}$ T. Yokoyama, Phys. Rev. B 77, 073413 (2008).

${ }^{30}$ Y. W. Son, M. L. Cohen, and S. G. Louie, Nature (London) 444, 347 (2006).

${ }^{31}$ O. V. Yazyev and M. I. Katsnelson, Phys. Rev. Lett. 100, 047209 (2008)
${ }^{32}$ M. Fujita, K. Wakabayashi, K. Nakada, and K. Kusakabe, J. Phys. Soc. Jpn. 65, 1920 (1996).

${ }^{33}$ J. Fernández-Rossier and J. J. Palacios, Phys. Rev. Lett. 99, 177204 (2007).

${ }^{34}$ T. Wassmann, A. P. Seitsonen, A. M. Saitta, M. Lazzeri, and F. Mauri, Phys. Rev. Lett. 101, 096402 (2008).

${ }^{35}$ H. Eisele and G. I. Haddad, Modern Semiconductor Device Physics, edited by S. M. Sze (Wiley, New York, 1998).

${ }^{36}$ T. Rakshit, G.-C. Liang, A. W. Ghosh, and S. Datta, Nano Lett. 4, 1803 (2005).

${ }^{37}$ I.-W. Lyo and Ph. Avouris, Science 245, 1369 (1989).

${ }^{38}$ H. Dalgleish and G. Kirczenow, Phys. Rev. B 73, 245431 (2006).

${ }^{39}$ V. M. García-Suárez and C. J. Lambert, Nanotechnology 19, 455203 (2008).

${ }^{40}$ L. Chen, Z. Hu, A. Zhao, B. Wang, Y. Luo, J. Yang, and J. G. Hou, Phys. Rev. Lett. 99, 146803 (2007).

${ }^{41}$ J. M. Soler, E. Artacho, J. D. Gale, A. García, J. Junquera, P. Ordejón, and D. Sánchez-Portal, J. Phys.: Condens. Matter 14, 2745 (2002).

${ }^{42}$ D. M. Ceperley and B. J. Alder, Phys. Rev. Lett. 45, 566 (1980).

${ }^{43}$ G. Román-Pérez and J. M. Soler, Phys. Rev. Lett. 103, 096102 (2009).

${ }^{44}$ A. R. Rocha, V. M. García-Suárez, S. W. Bailey, C. J. Lambert, J. Ferrer, and S. Sanvito, Phys. Rev. B 73, 085414 (2006).

${ }^{45}$ K. Nakada, M. Fujita, G. Dresselhaus, and M. S. Dresselhaus, Phys. Rev. B 54, 17954 (1996).

${ }^{46}$ E. H. Lieb, Phys. Rev. Lett. 62, 1201 (1989).

${ }^{47}$ J. Ferrer and V. M. García-Suárez, Phys. Rev. B 80, 085426 (2009).

${ }^{48}$ D. J. Adams, O. Gröning, C. A. Pignedoli, P. Ruffieux, R. Fasel, and D. Passerone, e-print arXiv:1201.4735v1. 